\title{
DETERMINACIÓN DEL GRADO DE METAPLASIA ESCAMOSA DEL EPITELIO CORNEAL COMO FACTOR DIAGNÓSTICO DE INSUFICIENCIA LIMBAL
}

\author{
CORNEAL EPITHELIUM SQUAMOUS METAPLASIA \\ DETERMINATION AS DIAGNOSTIC FACTOR IN LIMBAL \\ DEFICIENCY
}

\author{
LÓPEZ-GARCÍA JS ${ }^{1}$, RIVAS L ${ }^{2}$, GARCÍA-LOZANO I ${ }^{3}$
}

\begin{abstract}
RESUMEN
Objetivos: Determinar si existe relación entre el grado de metaplasia escamosa de la superficie corneal mediante citología de impresión y la gravedad clínica en pacientes con insuficiencia limbal.

Métodos: Hemos estudiado mediante citología de impresión 98 ojos de pacientes con insuficiencia limbal que fueron divididos en cuatro grupos según la gravedad clínica: 14 ojos no presentaban sintomatología alguna, 34 ojos presentaron un grado leve de insuficiencia limbal, 28 ojos un grado moderado y 22 ojos una insuficiencia limbal grave. A todos los pacientes se les realizó una citología corneal en la que se estudió el tamaño celular, tamaño nuclear, alteraciones nucleares y relación núcleo-citoplasma (N:C), así como la presencia de células caliciformes.

Resultados: Los pacientes con insuficiencia limbal asintomática presentaron un tamaño celular medio de 477 DE $140 \mu \mathrm{m}^{2}$, con una relación N:C de 1:5,25 DE 1,5. Los pacientes con insuficiencia limbal leve presentaron un tamaño celular medio de $764 \mathrm{DE}$ $122,6 \mu \mathrm{m}^{2}$ y una relación $\mathrm{N}: \mathrm{C}$ de 1:8,2 DE 1,4. Nin-
\end{abstract}

\begin{abstract}
Purpose: To determine the correlation between the squamous metaplasia grade of the corneal surface and the clinical severity of the limbal deficiency in patients with this disorder.

Methods: We studied 98 eyes of patients with limbal deficiency by impression cytology. These patients were divided into four groups in relation to their clinical severity: 14 eyes had no symptoms, 34 eyes showed a mild grade of limbal deficiency, 28 eyes had a moderate grade and 22 eyes had severe limbal deficiency. Corneal cytology was performed in each patient. Cellular size, nuclear size, nuclear changes and the nuclear-cytoplasmic $(\mathrm{N}: \mathrm{C})$ ratio were defined in corneal epithelial cells, in addition to evaluation for the presence of goblet cells in the corneal epithelium.

Results: In patients with limbal deficiency without symptoms, we found that the cellular size was 477 (SD140) $\mu \mathrm{m}^{2}$ and the $\mathrm{N}: \mathrm{C}$ ratio was 1:5.25 (SD1.5). In patients with mild limbal deficiency, the cellular size was 764 (SD122.6) $\mu \mathrm{m}^{2}$ and the $\mathrm{N}: \mathrm{C}$ ratio was 1:8.2 (SD1.4). These patients did not show corneal
\end{abstract}

Recibido: 8/11/05. Aceptado: 18/5/06

1 Doctor en Medicina. Hospital Central de Cruz Roja. Madrid.

2 Doctor en Biología. Hospital Ramón y Cajal. Madrid.

3 Licenciada en Medicina. Instituto de Ciencias Visuales (INCIVI). Madrid.

Correspondencia:

J.S. López García

Servicio de Oftalmología

Hospital Central de Cruz Roja

Avenida Reina Victoria, 26

28003 Madrid

España

E-mail: docsantilopez@hotmail.com 
guno de estos pacientes presentó conjuntivalización del epitelio corneal. Los pacientes con insuficiencia limbal moderada presentaron un tamaño celular medio de 1.162 DE $340 \mu \mathrm{m}^{2}$ y una relación $\mathrm{N}: \mathrm{C}$ de 1:13,2 DE 3,6. Estos pacientes presentaron con cierta frecuencia conjuntivalización del epitelio corneal, definida por la presencia de células caliciformes. Los pacientes con insuficiencia limbal grave presentaron un tamaño celular medio de $2.036 \mathrm{DE}$ $382 \mu \mathrm{m}^{2}$ y una relación $\mathrm{N}: \mathrm{C}$ de 1:23,6 DE 4,0. En estos pacientes la presencia de conjuntivalización en la citología corneal fue la norma.

Conclusiones: Existe una correlación entre la gravedad clínica de la insuficiencia limbal y el grado de metaplasia escamosa determinado mediante citología de impresión corneal. La citología de impresión es particularmente útil para el diagnóstico en los casos de déficit limbal leve o subclínico.

Palabras claves: Aniridia. Deficiencia limbal. Citología de impresión. Superficie ocular. Quemaduras oculares. conjunctivalization. In patients with moderate limbal deficiency the cellular size was 1,162 (SD340.2) $\mu \mathrm{m}^{2}$ and the $\mathrm{N}: \mathrm{C}$ ratio was $1: 13.2$ (SD3.6). Some patients showed corneal conjunctivalization that was defined by the presence of goblet cells in the corneal epithelium. In patients with severe limbal deficiency, the cellular size was 2,036 (SD382) $\mu \mathrm{m}^{2}$ and the $\mathrm{N}: \mathrm{C}$ ratio was 1:23.6 (SD4.0). Corneal conjunctivalization was present in all of these patients. Conclusions: There was a correlation between limbal deficiency severity and squamous metaplasia grade defined by impression cytology. Impression cytology was very useful for the diagnosis of cases of mild or subclinical limbal deficiency (Arch Soc Esp Oftalmol 2006; 81: 281-288).

Key words: Aniridia, limbal deficiency, impression cytology, ocular surface, ocular burns.

\section{INTRODUCCIÓN}

El limbo presenta una gran importancia en la regeneración epitelial de la córnea pues a este nivel se encuentran las células madre progenitoras del epitelio corneal. Estas células, clásicamente localizadas en las empalizadas de Vogt (1), presentan una serie de propiedades comunes a otras células madre localizadas a otros niveles como la pluripontecialidad, la capacidad de autorrenovación, su larga vida y un ciclo celular prolongado $(2,3)$. Hasta el momento, no existen marcadores específicos de estas células madre limbares siendo únicamente demostrables por evidencias indirectas (4).

La insuficiencia límbica es una entidad clínica que se caracteriza por una vascularización del epitelio corneal, inflamación crónica, defectos epiteliales persistentes y recurrentes, fotofobia, ojo rojo y pérdida de visión (5). Está producida por una disfunción de las células progenitoras del epitelio corneal que puede ser total o parcial dependiendo del grado de afectación de estas células madre.

Esta disfunción limbal puede ocurrir como consecuencia de una destrucción del limbo por agresiones externas como ocurre en el caso de quemaduras oculares, portadores de lentes de contacto, múltiples cirugías sobre el área limbal, aplicación de crioterapia o tras infecciones microbianas graves. También puede observarse asociada a dermopatías como el penfigoide ocular cicatricial, síndrome de Stevens-Johnson, Síndrome de Lyell, rosacea, etc. Con menor frecuencia se asocia a enfermedades congénitas como la aniridia o presenta un carácter idiopático (6-8).

La citología de impresión nos permite estudiar las capas celulares más superficiales del epitelio córneal y conjuntival, pudiendo diagnosticar un considerable número de trastornos de la superficie ocular (9-11). Aunque la aplicación más común de la citología de impresión en la práctica clínica oftalmológica es valorar el grado de metaplasia escamosa conjuntival en pacientes con clínica de ojo seco (12), ésta también ha sido utilizada para valorar el grado de recuperación de la superficie corneal en pacientes tratados con trasplante de membrana amniótica o cirugía combinada de ésta con trasplante de limbo (13-15).

En este trabajo pretendemos relacionar la gravedad clínica de la insuficiencia limbal con la severidad de la metaplasia escamosa del epitelio corneal estimada mediante citología de impresión, con el fin de determinar el valor diagnóstico de ésta en pacientes con trastornos de la superficie ocular secundarios a déficit limbal. 


\section{SUJETOS, MATERIAL Y MÉTODOS}

En la selección de pacientes, técnicas aplicadas y manejo de las muestras se cumplieron las normas nacionales e internacionales para estudios clínicos. A todos los pacientes que, de forma voluntaria, quisieron intervenir en este estudio se les solicitó por escrito la aceptación de su participación.

Hemos realizado un estudio sobre 98 ojos de 57 pacientes (35 hombres y 22 mujeres) con insuficiencia limbal causada por diversas etiologías: quemaduras oculares (24 ojos), aniridia (22 ojos), postcirugía de pterigion u otros procesos con implicación conjuntival (12), penfigoide ocular cicatricial (10), portadores de lentes de contacto (10), síndrome de Stevens-Johnson (6), síndrome de Lyell (4), acné rosácea (4), otros de causa desconocida (6). Mediante anamnesis y exploración se establecieron cuatro grupos clínicos dependiendo de que los pacientes no manifestaran trastornos clínicos relacionados con la deficiencia limbal (grupo 0) o estos fuesen leves (grupo 1), moderados (grupo 2) o graves (grupo 3). La distribución de los pacientes según etiologías en los distintos grupos clínicos viene reflejada en la tabla I.

Para establecer los grupos clínicos se valoró la gravedad de distintos signos y síntomas producidos por la deficiencia limbal. Se consideró que un paciente presentaba clínica de insuficiencia limbal leve (grupo 1) cuando el paciente refería un máximo de dos episodios de úlceras recurrentes o erosiones recidivantes en los últimos 6 meses y leve fotofobia y epífora. A la exploración con lámpara de hendidura, estos pacientes no presentaban alteraciones significativas, salvo que coincidiese con algún periodo de actividad. El pannus, cuando existía, era leve y no pasaba más de $1 \mathrm{~mm}$ de la arcada límbica y mostraban leves trastornos en la captación de fluoresceína. En este grupo se incluyeron 34 ojos. Se consideró que un paciente presentaba clínica de insuficiencia limbal moderada (grupo 2) cuando el número de episodios de erosiones recidivantes o úlceras recurrentes fue igual o superior a 3 episodios en los últimos 6 meses y en la exploración con lámpara de hendidura se observaba una inestabilidad permanente de la película lagrimal y un pannus vascular, acompañado o no de tejido fibroso subepitelial, que afectaba a menos de la mitad periférica de la córnea (fig. 1). La fotofobia, epífora y ojo rojo fueron constantes. En este grupo se incluyeron 28 ojos. Se consideró que un paciente presentaba clínica de insuficiencia limbal grave (grupo 3) cuando en la exploración con lámpara de hendidura el paciente presentaba una vascularización corneal que afectaba al centro de la córnea, así como clínica de erosiones corneales e inestabilidad de la película lagrimal permanente (figs. 2 y 3 ). $\mathrm{La}$ fotofobia, epífora y ojo rojo fueron la norma, así como la pérdida de visión por afectación del eje visual. En este grupo se incluyeron 22 ojos. En el grupo " 0 " incluimos a 14 ojos de pacientes con procesos etiológicos susceptibles de insuficiencia limbal que no manifestaban clínica relacionada con ella (fig. 4).

El estudio de la superficie corneal mediante citología de impresión se realizó obteniendo muestras

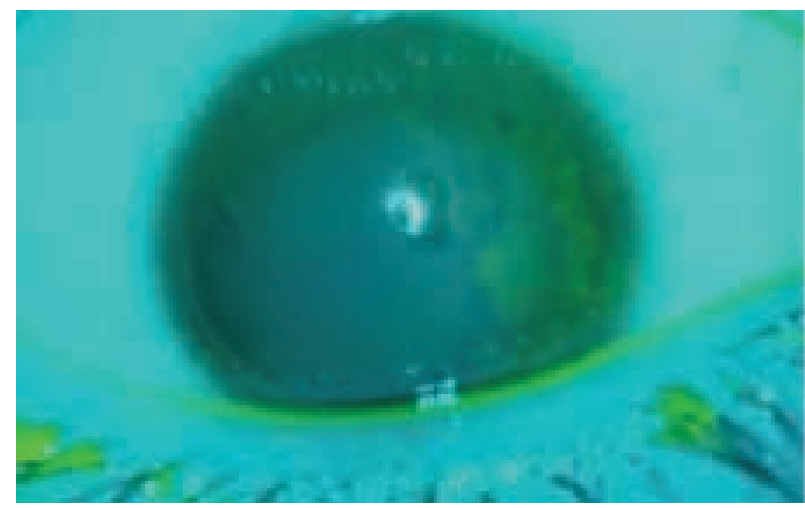

Fig. 1: Trastornos en la captación de fluoresceína en la periferia de un paciente con insuficiencia limbal moderada.

Tabla I. Distribución de los ojos según etiología y gravedad clínica

\begin{tabular}{lrcccccccc}
\hline & Q. O & aniridia & Postc. & POC & PLC & SSJ & S.Lyell & A.R & Otros \\
\hline Grupo 0 & 0 & 4 & 2 & 0 & 6 & 0 & 0 & 0 & 2 \\
Grupo 1 & 5 & 10 & 8 & 0 & 4 & 0 & 0 & 3 & 4 \\
Grupo 2 & 12 & 4 & 2 & 4 & 0 & 4 & 1 & 1 & 0 \\
Grupo 3 & 7 & 4 & 0 & 6 & 0 & 2 & 3 & 0 & 0 \\
\hline \hline
\end{tabular}

Q.O: quemaduras oculares; Post. Postcirugía; POC: penfigoide ocular cicatricial; PLC: portador de lentes de contacto; SSJ: síndrome de StevensJohnson; A.R: acné rosacea. 


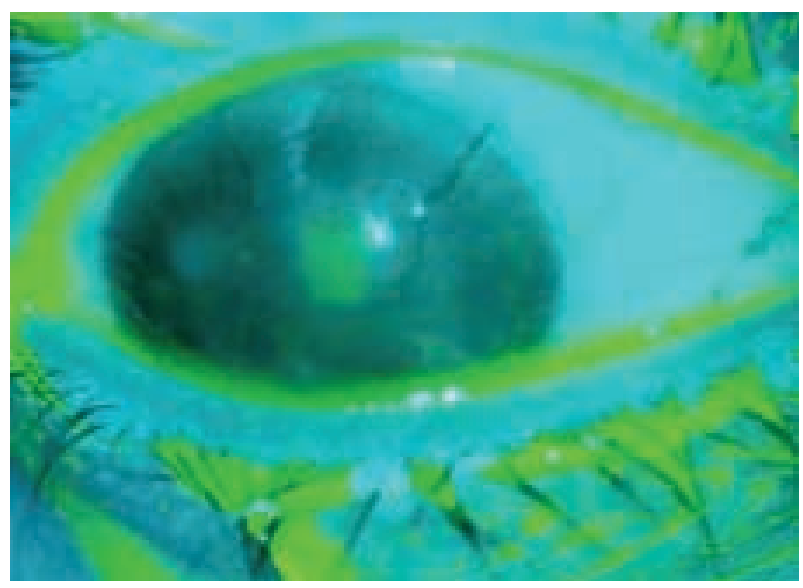

Fig. 2: Úlcera central persistente en un paciente con insuficiencia limbal grave. Se puede apreciar como la vascularización afecta al centro de la córnea.

de los cuatro cuadrantes recogidas sobre tiras de papel Millipore HAWP304 de 5x5 mm de tamaño, fijadas con etanol al 96\% durante 10 minutos y teñidas con PAS-hematoxilina de acuerdo con el protocolo de Locquin y Langeron modificado por Rivas et al. (16), observándose posteriormente con microscopio óptico.

Para el estudio morfométrico de estas células se trabajó a 40 aumentos sobre fotografías de 10x15 $\mathrm{cm}$. Se empleó un equipo de análisis de imagen compuesto por un microscopio Nikon Ophtiphot-2 dotado de equipo fotográfico Microflex-DX y de una videocámara en color EVI-10011P acoplada a un monitor Sony Black Trinitron KT-14 CP1. El sistema está basado en el software IPP-Plus de Image Pro/Media Cibermetics y el sistema CAD.

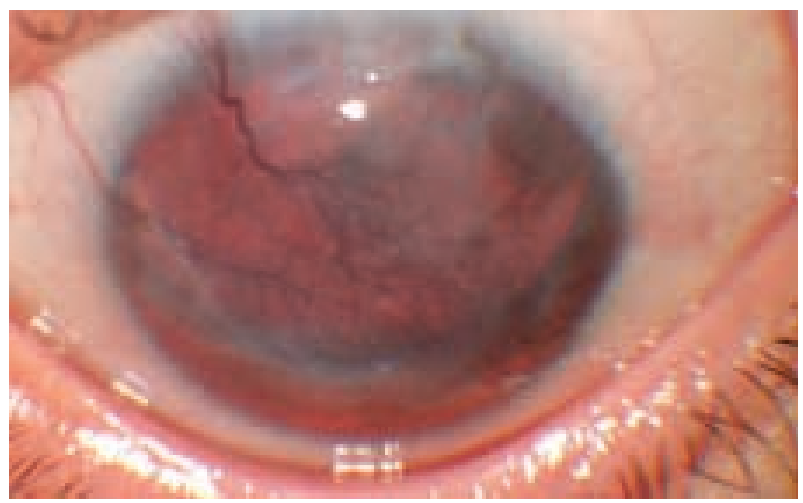

Fig. 3: Insuficiencia limbal grave en un paciente con aniridia congénita.

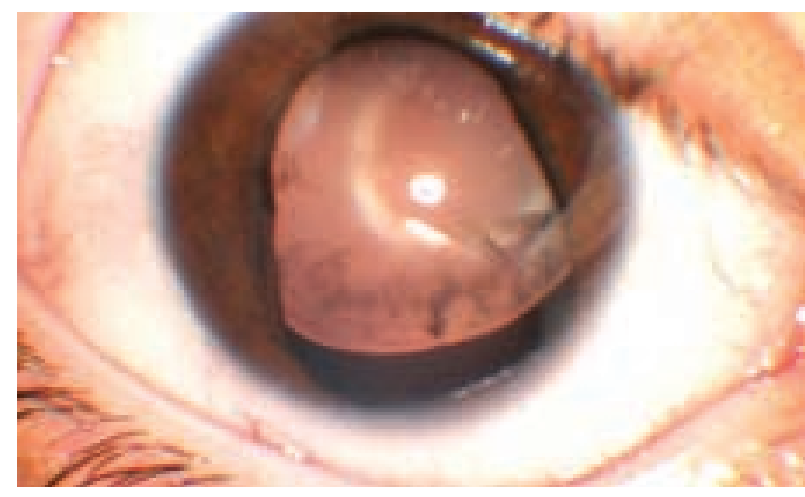

Fig. 4: Paciente con aniridia congénita sin clínica relacionada con la deficiencia limbal.

Para valorar el grado de alteración celular utilizamos la clasificación de metaplasia escamosa propuesta por Murube y Rivas (17). Esta clasificación distingue 6 grados de metaplasia, donde el grado 0 representa la normalidad, atendiendo a criterios como la forma y tamaño de las células, la separación entre ellas, su tamaño nuclear, la tinción del citoplasma, las alteraciones nucleares, la relación núcleo-citoplasma $(\mathrm{N}: \mathrm{C})$ y la conjuntivalización del epitelio corneal.

El análisis estadístico se realizó mediante el programa informático SPSS versión 9.0 para Windows. Las medidas utilizadas fueron la media y desviación estándar. Los resultados entre distintos grupos fueron comparados utilizando el test ANOVA unifactorial con corrección posterior mediante el test HSD de Tukey de comparaciones múltiples para una significación estadística $\mathrm{p}<0,05$. El grado de correlación fue determinado mediante el test Rho de Spearman.

\section{RESULTADOS}

En los pacientes sin clínica de insuficiencia limbal (grupo 0), encontramos en la citología de impresión unas células epiteliales con un tamaño celular medio de $477 \mathrm{DE} 140 \mu^{2}$, un tamaño nuclear medio de 92 DE 3,13 $\mu \mathrm{m}^{2}$ y una relación N:C de 1:5 DE 1,5. Las muestras citológicas presentaban abundante celularidad, con células muy homogéneas en cuanto al tamaño y con pocos espacios intercelulares. En todos los casos la tinción del citoplasma fue eosinófila y la presencia de alteraciones nucleares fue inferior a un $10 \%$ de los casos, siendo la pre- 
sencia de núcleos serpentiformes la alteración nuclear observada más frecuentemente. No encontramos células caliciformes en ninguno de los pacientes de este grupo. Salvo 4 casos, que presentaron un grado de metaplasia grado 2 , el resto de los ojos presentó una metaplasia grado 1 .

El grupo de pacientes con insuficiencia limbal leve (grupo 1) presentó una metaplasia escamosa grado 2. Las células del epitelio corneal tuvieron un tamaño celular medio de 764 DE $122 \mu^{2}$, un tamaño nuclear de $91 \mathrm{DE} 2,7 \mu \mathrm{m}^{2}$ y una relación $\mathrm{N}: \mathrm{C}$ de 1:8 DE 1,4, presentando pocos espacios intercelulares entre ellas. Salvo algún caso, la tinción del citoplasma fue eosinófila. En menos de un $30 \%$ de los casos encontramos alteraciones nucleares, siendo las más comunes la presencia de células binucleadas y núcleos serpentiformes. En ninguno de estos pacientes encontramos presencia de células caliciformes en la citología corneal (fig. 5).

En el grupo de pacientes con insuficiencia limbal moderada (grupo 2), las células del epitelio corneal presentaron un tamaño celular medio de $1162 \mathrm{DE}$ $340 \mu \mathrm{m}^{2}$, un tamaño nuclear de $87 \mathrm{DE} 2,5 \mu \mathrm{m}^{2}$ y una relación N:C de 1:13 DE 3,6. Las células epiteliales presentaron una mayor dispersión en cuanto al tamaño celular, siendo menor la celularidad de las muestras y aumentando considerablemente la presencia de espacios intercelulares. La tinción predominante del citoplasma fue eosinófila pero en algunos casos esta presentó un aspecto metacromá-

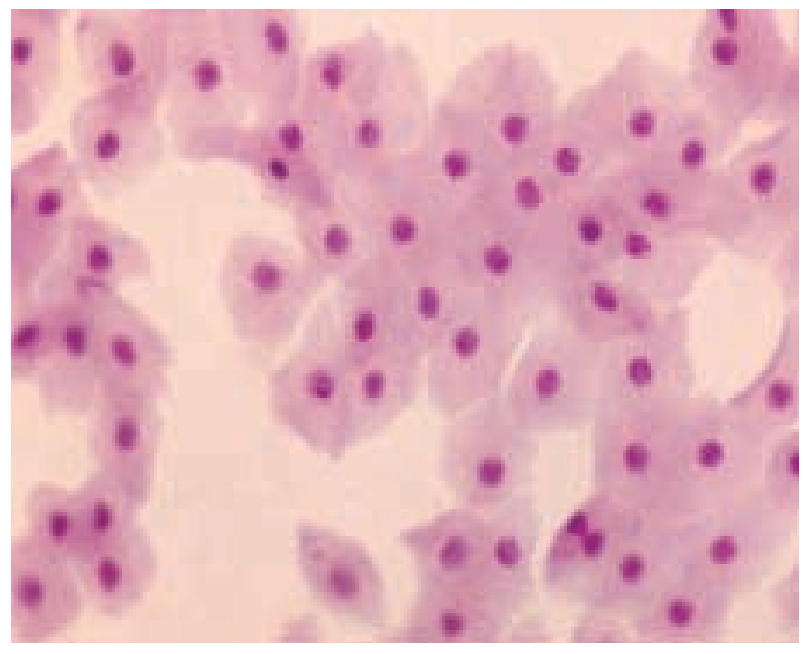

Fig. 5: Citología de impresión corneal en un paciente con una insuficiencia limbal leve. Se aprecian unas células homogéneas, sin alteraciones nucleares importantes, con citoplasma eosinófilo y una relación $N: C$ de 1:8. tico. Encontramos alteraciones nucleares en más de un $40 \%$ de los casos, siendo las más comunes la presencia de células binucleadas, núcleos serpentiformes y células con núcleos picnóticos. Considerando todos estos parámetros, estas células presentaron una metaplasia escamosa grado 2-3. En este grupo de pacientes encontramos en un $25 \%$ de los casos conjuntivalización corneal que vino definida por la presencia, aunque en escaso número, de células caliciformes con pobre contenido mucínico en la citología corneal (fig. 6).

En el grupo de pacientes con insuficiencia limbal grave (grupo 3), encontramos unas células epiteliales con un tamaño medio de $2036 \mathrm{DE} 382 \mu \mathrm{m}^{2}$, un tamaño nuclear de $82 \mathrm{DE} 2,2 \mu \mathrm{m}^{2}$ y una relación $\mathrm{N}: C$ de 1:23 DE 4. Las células mostraron importantes diferencias de tamaños entre ellas, con grandes y abundantes espacios intercelulares e incluso signos de queratinización. La tinción del citoplasma fue predominantemente metacromática, aunque fue relativamente común la presencia de células con citoplasma basófilo. La presencia de alteraciones nucleares fue la norma encontrándolas en más del $75 \%$ de los pacientes. La alteración nuclear más común fue, con mucho, la presencia de núcleos picnóticos, seguido por la presencia de células anucleadas y células binucleadas. La mayoría de los pacientes, presentaron una metaplasia escamosa grado 4, aunque en este grupo también encontramos pacientes con metaplasia escamosa grados 3 y 5 . La presencia en mayor o menor número de células cali-

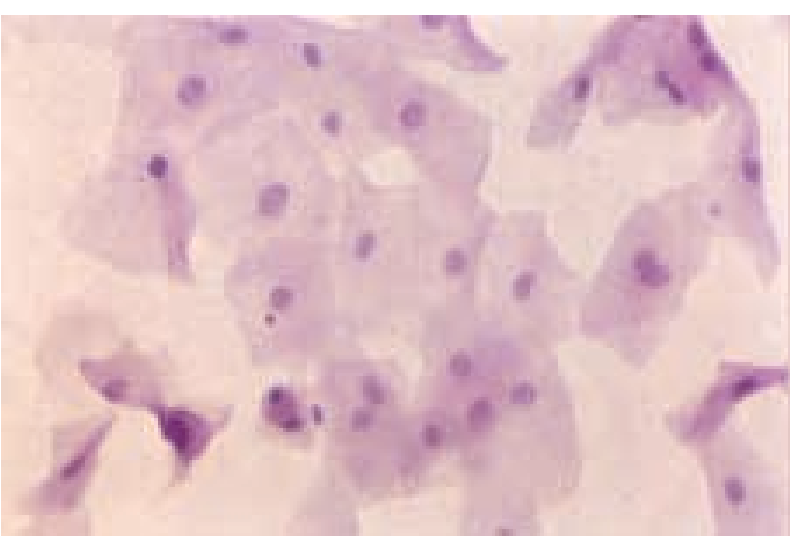

Fig. 6: Citología de impresión corneal en un paciente con insuficiencia limbal moderada. Se aprecia una menor celularidad, con alteraciones nucleares frecuentes como células binucleadas y una tinción metacromática del citoplasma, así como la presencia de células caliciformes. 
ciformes en la citología corneal fue la norma en la totalidad de los pacientes de este grupo (fig. 7).

En la tabla II quedan recogidos los valores de la variables cuantitativas determinados mediante citología de impresión en los distintos grupos clínicos. Encontramos diferencias estadísticamente significativas en cuanto al tamaño celular, relación $\mathrm{N}$ :C y grado de metaplasia escamosa entre todos los grupos clínicos, mientras que en cuanto al tamaño

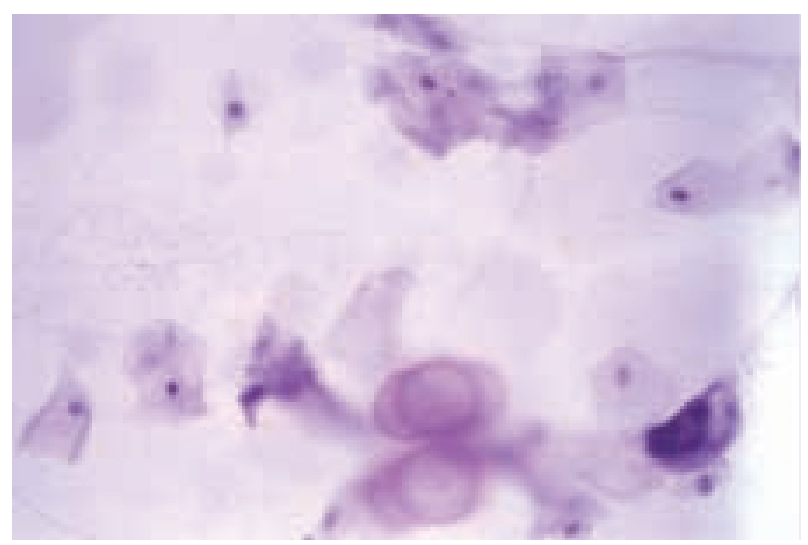

Fig. 7: Citología de impresión corneal en un paciente con insuficiencia limbal grave. Se aprecian células muy heterogéneas con núcleos picnóticos o anucleadas y citoplasma basófilo. Destaca también la presencia de células caliciformes.

Tabla II. Relación de los valores medios de los parámetros cuantitativos obtenidos en los distintos grupos clínicos (media y desviación estándar)

\begin{tabular}{lrlll}
\hline & T. celular* & T. nuclear* & Rel. N:C & Metaplasia \\
\hline Grupo 0 & $477 \mathrm{DE} 140$ & $92,5 \mathrm{DE} 3,1$ & $5,25 \mathrm{DE} 1,5$ & $1,25 \mathrm{DE} 0,45$ \\
Grupo 1 & 764DE122 & $91,0 \mathrm{DE} 2,7$ & $8,26 \mathrm{DE} 1,4$ & $1,93 \mathrm{DE} 0,25$ \\
Grupo 2 & 1162DE340 & 87,2DE2,5 & $13,2 \mathrm{DE} 3,6$ & $2,61 \mathrm{DE} 0,7$ \\
Grupo 3 & $2036 \mathrm{DE} 382$ & $82,6 \mathrm{DE} 2,2$ & $23,6 \mathrm{DE} 4,0$ & $4,18 \mathrm{DE} 0,4$ \\
\hline \hline
\end{tabular}

(*) Micras². nuclear éstas lo fueron sólo en los casos de insuficiencia limbal moderada y grave (grupos 2 y 3 ). Por otro lado, encontramos una correlación significativa al nivel 0,01 bilateral mediante el test Rho de Spearman entre la gravedad clínica de la insuficiencia limbal y el grado de metaplasia escamosa determinado mediante citología de impresión. Encontramos una correlación negativa entre la gravedad clínica y el tamaño nuclear, mientras que esta fue positiva con el tamaño celular y la relación $\mathrm{N}$ :C (tabla III).

\section{DISCUSIÓN}

La metaplasia escamosa es un mecanismo de respuesta adaptativa, a menudo reversible, de algunos epitelios ante agresiones externas o estímulos patogénicos del propio organismo. Este grado de metaplasia nos informa del estado de la superficie ocular y, por tanto, de la severidad de la enfermedad. Aunque son muchas las aplicaciones de la citología de impresión como prueba diagnóstica (11), la principal aplicación en la práctica clínica habitual es el estudio de la metaplasia del epitelio tanto corneal como, sobre todo, conjuntival (17).

Las alteraciones a nivel del limbo producen importantes trastornos a nivel del la superficie del epitelio corneal que pueden ser detectados mediante la citología de impresión (18). En este trabajo planteamos un estudio trasversal para determinar las características citológicas del epitelio corneal en diferentes entidades etiológicas que tienen en común la presencia de un cuadro de deficiencia limbal. Sin embargo, esta diversidad etiológica nos ha de hacer ser prudentes a la hora de generalizar los resultados obtenidos, pues no en todas ellas el daño limbal se produce por los mismos mecanismos patogénicos (19-21). De igual forma, la mayor o menor cronicidad del cuadro clínico influye en el grado de respuesta reactiva del epitelio corneal y

Tabla III. Coeficiente de correlación estimado mediante el test Rho de Spearman. En todos los casos la correlación es significativa a nivel 0,01

\begin{tabular}{lccccc}
\hline & Gravedad & T. celular & T. nuclear & Rel. N:C & Metaplasia \\
\hline Gravedad & &, 871 &,- 779 &, 906 &, 864 \\
T. celular &, 871 &,- 682 &,- 682 &, 988 &, 923 \\
T. nuclear &,- 779 &, 988 &,- 733 &,- 726 \\
Rel. N:C &, 906 &, 923 &,- 733 &, 932 \\
Metaplasia &, 864 &,- 726 &, 932 &, 932 \\
\hline \hline
\end{tabular}


hemos de tener presente que esta respuesta no se produce sólo por la insuficiencia limbal. Según estas consideraciones, y para tratar de homogeneizar lo máximo posible la muestra, hemos clasificado a los pacientes atendiendo fundamentalmente a parámetros clínicos derivados del grado de deficiencia limbal, sin tener en cuenta otros signos o síntomas característicos de cada entidad.

En este trabajo encontramos una correlación entre la gravedad clínica de la insuficiencia limbal y las alteraciones morfológicas observadas en las células del epitelio corneal mediante citología de impresión. Encontramos un mayor grado de metaplasia escamosa a mayor gravedad del cuadro clínico. Así, pacientes con una insuficiencia limbal grave (grupo 3) presentaron de media una metaplasia grado 4 , mientras que pacientes con una insuficiencia limbal leve (grupo 2) presentaron una metaplasia grado 2. Si analizamos por separado los distintos parámetros estudiados mediante la citología de impresión, vemos como son parámetros cuantitativos como el tamaño celular y la relación N:C en los que esta correlación es particularmente llamativa, además estos dos parámetros presentaron diferencias estadísticamente significativas entre todos los grupos clínicos. Por otro lado, hemos encontrado como pacientes que no presentaban clínica de insuficiencia limbal (grupo 0) tenían, en algunos casos, una metaplasia escamosa grado 2. Según estos datos, la citología de impresión nos permitiría poder diagnosticar la insuficiencia limbal incluso antes de que esta fuese clínicamente evidente. En este sentido, hemos de decir que el diagnóstico de insuficiencia limbal es fácil en los casos moderados y graves, sobre todo si existe un antecedente traumático. Sin embargo, en los casos leves de déficit limbal este diagnóstico puede pasar fácilmente desapercibido. En estos casos con afectación leve o preclínica, la citología de impresión al igual que la fluorofotometría puede ser de gran utilidad diagnóstica (22).

Según nuestros resultados, podemos concluir diciendo que existe una correlación entre la gravedad clínica de la insuficiencia limbal y el grado de metaplasia escamosa determinado mediante citología de impresión corneal. Asimismo, la citología de impresión nos ayuda a establecer el diagnóstico de deficiencia limbal en casos leves y subclínicos y nos permite monitorizar los cambios producidos en la superficie corneal por efecto del tratamiento.

\section{BIBLIOGRAFÍA}

1. Davanger M, Evensen A. Role of the pericorneal papillary structure in renewal of corneal epithelium. Nature 1971; 229: 560-561.

2. Dua HS, Azuara-Blanco A. Limbal stem cells of the corneal epithelium. Surv Ophthalmol 2000; 44: 415-425.

3. Beebe DC, Masters BR. Cell lineage and the differentiation of corneal epithelial cells. Invest Ophthalmol Vis Sci 1996; 37: 1815-1825.

4. Wolosin JM, Schutte M, Zieske JD, Budack MT. Changes in connexin43 in early ocular surface development. Curr Eye Res 2002; 24: 430-438.

5. Tseng SC. Regulation and clinical implications of corneal epithelial stem cells. Mol Biol Rep 1996; 23: 47-58.

6. Nghiem-Buffet MH, Gatinel D, Jacquot F, Chaine G, Hoang-Xuan T. Limbal stem cell deficiency following phototherapeutic keratectomy. Cornea 2003; 22: 482484.

7. Tsai RJ, Tseng SC. Effect of stromal inflammation on the outcome of limbal transplantation for corneal surface reconstruction. Cornea 1995; 14: 439-449.

8. Espana EM, Grueterich M, Romano AC, Touhami A, Tseng SC. Idiopathic limbal stem cell deficiency. Ophthalmology 2002; 109: 2004-2010.

9. Rivas L, Oroza MA, Perez-Esteban A, Murube-del-Castillo J. Morphological changes in ocular surface in dry eyes and other disorders by impression cytology. Graefes Arch Clin Exp Ophthalmol 1992; 230: 329-334.

10. Sridhar MS, Vemuganti GK, Bansal AK, Rao GN. Impression cytology-proven corneal stem cell deficiency in patients after surgeries involving the limbus. Cornea 2001; 20: 145-148.

11. McKelvie P. Ocular surface impression cytology. Adv Anat Pathol 2003; 10: 328-337.

12. Murube J, Rivas L. Byopsy of the conjunctiva in dry eye patients establishes a correlation between squamous metaplasia and dry eye clinical severity. Eur J Ophthalmol 2003; 13: 246-256.

13. Lopez-Garcia JS, Rivas L, Garcia-Lozano I. Trasplante de membrana amniótica en el tratamiento de la insuficiencia limbal moderada de pacientes con aniridia congénita. Arch Soc Esp Oftalmol 2005; 80:517-523.

14. Lopez-Garcia JS, Rivas L, Garcia-Lozano I. Tratamiento de la insuficiencia limbal grave mediante cirugía combinada de trasplante de limbo y trasplante de membrana amniótica. Arch Soc Esp Oftalmol 2005; 80: 405-412.

15. Prabhasawat P, Tseng SC. Impression cytology study of epithelial phenotype of ocular surface reconstructed by preserved human amniotic membrane. Arch Ophthalmol 1997; 115: 1360-1367.

16. Rivas L, Oroza MA, Perez-Esteban A, Murube-del-Castillo J. Topographical distribution of ocular surface cells by the use of impression cytology. Acta Ophthalmol 1991; 69: 371-376.

17. Murube J, Rivas L. Impression cytology on conjunctiva and cornea in dry eye patients establishes a correlation between squamous metaplasia and dry eye clinical severity. Eur J Ophthalmol 2003; 13: 115-127.

18. Donisi PM, Rama P, Fasolo A, Ponzin D. Analysis of limbal stem cell deficiency by corneal impression cytology. Cornea 2003; 22: 533-538. 
19. Tsubota K, Satake Y, Ohyama M, Toda I, Takano Y, Ono $M$, et al. Surgical reconstruction of the ocular surface in advanced ocular cicatricial pemphigoid and StevensJohnson syndrome. Am J Ophthalmol 1996; 122: 38-52.

20. Rivas L, Murube J, Rivas A, Murube E. Estudio del ojo seco en pacientes con aniridia congénita, mediante citología de impresión. Arch Soc Esp Oftalmol 2003; 78: 615 622.
21. Reim M, Redbrake C, Schrage N. Heridas oculares químicas y térmicas. Tratamiento quirúrgico y médico basado en hallazgos clínicos y patofisiológicos. Arch Soc Esp Oftalmol 2001; 76: 79-124.

22. Kinoshita S, Adachi W, Sotozono C, Nishida K, Yokoi N, Quantock AJ, et al. Characteristics of the human ocular surface epithelium. Prog Retin Eye Res 2001; 20: 639673. 